\title{
Effect of Preexcisional Heparin Anticoagulation on Excised Canine Vein and Artery Prostanoid Production
}

\author{
Timothy F. KresowiK, M.D., Christopher K. Vincent, M.D., Jan S. Brunkwall, M.D., \\ AND JAMES C. STANLEY, M.D. \\ Departments of Surgery, University of Iowa College of Medicine, Iowa City, Iowa, \\ and University of Michigan Medical School, Ann Arbor, Michigan
}

Presented at the Annual Meeting of the Association for Academic Surgery, Salt Lake City, Utah, November 16-19, 1988

\begin{abstract}
The effect of heparin, administered prior to vessel dissection and excision, on the luminal release of prostanoids from an excised vessel was assessed. Eight adult mongrel dogs underwent removal of the jugular vein and carotid artery on one side, followed by intravenous administration of heparin sodium (150 IU/kg) and subsequent removal of these same vessels from the contralateral neck. The excised vessels were perfused in an ex vivo system with Hanks' balanced salt solution for five consecutive 15-min periods. Prostacyclin release (measured as 6keto-PGF ${ }_{1} \alpha$ ) and thromboxane $A_{2}$ (measured as thromboxane $B_{2}$ ) release into the perfusate were quantitated by radioimmunoassay. Vessel segments were studied with and without first period thrombin stimulation (2 $\mathrm{U} / \mathrm{ml})$ and with arachidonic acid $(4 \mu \mathrm{g} / \mathrm{ml})$ stimulation during the last perfusion period. Vein segments following heparin administration exhibited greater prostacyclin production than veins not exposed to heparin. This effect did not occur in arteries. Heparin did not influence thromboxane $A_{2}$ release. Luminal endothelial cell coverage was not affected by the presence or absence of heparin. Thus heparin anticoagulation prior to dissection and excision of a vein, may enhance early preservation of its endothelial cell function as evident by increased luminal release of prostacyclin. (c) 1989 Academic Press, Inc.
\end{abstract}

\section{INTRODUCTION}

Autogenous blood vessels are preferred conduits for most small vessel reconstructions. The higher patency of such grafts compared to prosthetic grafts, especially in low-flow situations, has been attributed in part to the antithrombotic properties of endothelium. Endothelial cells are the major source of prostacyclin $\left(\mathrm{PGI}_{2}\right)$, a powerful vasodilator and antagonist of platelet aggregation. The prostaglandin thromboxane $\mathrm{A}_{2}\left(\mathrm{TxA}_{2}\right)$, a vasoconstrictor and a stimulus for platelet aggregation, has as its primary source platelets, although some $\mathrm{TxA}_{2}$ is produced by components of the vessel wall including endothelium $[1,2]$. A favorable balance between $\mathrm{PGI}_{2}$ and $\mathrm{TxA}_{2}$ may be important in maintaining vessel patency.
Certain failures of autogenous artery and vein grafts have been attributed to endothelial damage occurring during procurement and preparation procedures. Excessive dissection, overzealous distension of the vessel, prolonged exposure to crystalloid solutions, and vasospasm have been implicated as causes of endothelial cell injury [3]. Thrombin or fibrin produced as a result of thrombin generation may also be harmful to endothelium $[4,5]$. Heparin, by facilitating the action of endogenous antithrombins, may inhibit generation of thrombin and thrombin-catalyzed conversion of fibrinogen to fibrin and thus protect the integrity of endothelial cells $[6,7]$. The purpose of this experiment was to document the effect of heparin administration prior to vessel harvest on endothelial cell function as manifest by prostanoid production.

\section{MATERIALS AND METHODS}

Vessel harvest. Eight adult mongrel dogs were anesthetized with pentobarbital and mechanically ventilated. Through a midline neck incision a carotid artery and ipsilateral external jugular vein were dissected. These vessels were excised independently and prepared for ex vivo perfusion. Heparin sulfate (150 units/kg) was then administered intravenously, and the contralateral carotid artery and jugular vein were harvested in a manner similar to that of the opposite side. These vessels were also perfused independently. Animal care was carried out in accordance with Principles of Laboratory Animal Care and Guide for The Care of and Use of Laboratory Animals (NIH publication No. 80-23, Revised, 1976).

Vessel perfusion. Each excised vein and artery was gently flushed immediately following excision with roomtemperature calcium- and magnesium-free Hanks' balanced salt solution (CMF-HBSS) (GIBCO, Grand Island, $\mathrm{NY)}$ which had been adjusted to $p \mathrm{H}$ 7.4. Vessels were then divided into two equal segments, $30-40 \mathrm{~mm}$ in length, and mounted in the perfusion system. Prior to the first perfusion the vessel segments were flushed with $25 \mathrm{ml}$ of CMF-HBSS to remove any prostaglandins released during the mounting process. The vessel segments were perfused 
with $25 \mathrm{ml}$ of HBSS (GIBCO, Grand Island, NY) adjusted to a $p \mathrm{H}$ of 7.4 at $37^{\circ} \mathrm{C}$ in a recirculating loop for five separate 15-min periods [8]. At the conclusion of each 15-min perfusion, the perfusate was collected and replaced with new HBSS. Perfusate samples were stored at $-20^{\circ} \mathrm{C}$ until subsequent prostanoid assays were performed.

Veins were perfused with nonpulsatile flow, created by placing a Windkessel device in the circuit, at a rate of 90 $\mathrm{ml} / \mathrm{min}$ and a pressure of $7 \mathrm{~mm} \mathrm{Hg}$. Arteries were perfused with pulsatile flow of $90 \mathrm{ml} / \mathrm{min}$ and a mean pressure of $100 \mathrm{~mm} \mathrm{Hg}$. Fifty units of human thrombin (T-7009, Sigma, St. Louis, MO) was added to the first perfusate in one segment from each vessel. Arachadonic acid $100 \mu \mathrm{g}$ (A-6382, Sigma, St. Louis, MO) was added to the last perfusate of all vessel segments tested.

Scanning electron microscopy. Vessel segments were perfusion-fixed after the prostanoid release studies in $2.5 \%$ glutaraldehyde with $0.1 \mathrm{M}$ cacodylate. The vessels were dehydrated in ethanol, air-dried after saturation with hexamethyldisilazane, and sputter-coated with gold-palladium. The percentage of luminal endothelial cell coverage was estimated by scanning electron microscopy. Five separate areas were examined on each vessel segment, being scored 0,1 , or 2 . A score of 0 corresponded to absence of endothelium and 2 represented an intact layer of endothelium. The sum of the scores of the five areas defined the surface coverage (sum $\times 10=$ percentage coverage).

Prostanoid analysis. Prostacyclin and thromboxane $\mathrm{A}_{2}$ production were determined by measuring their stable breakdown products 6 -keto- $\mathrm{PGF}_{1} \alpha$ and thromboxane $\mathrm{B}_{2}$ $\left(\mathrm{TxB}_{2}\right)$. These prostaglandins were extracted from the perfusate samples using a modification of the method described by Simmons and colleagues in which proteins were precipitated with acetonitrile and discarded, with the su- pernatant being acidified with formic acid prior to ethyl acetate extraction [9]. These samples were air-dried and redissolved in $0.1 \%$ gel phosphate-buffered saline. 6-keto$\mathrm{PGF}_{1} \alpha$ and $\mathrm{TxB}_{2}$ were measured with commercial RIA kits using a ${ }^{3} \mathrm{H}$ tracer (New England Nuclear, Boston, MA). The sample values were determined from a standard curve calculated for each run.

Data are expressed as means \pm 1 SEM. Statistical significance was determined with a paired $t$ test comparing the pre- and postheparin segments in the same animal.

\section{RESULTS}

Prostacyclin release from arteries was generally 5 to 10 times greater than from veins (Table 1, Figs. 1 and 2). Both arteries and veins exhibited a similar pattern of decreasing prostacyclin production during the 75 -min perfusion. Arachidonic acid addition to the last 15 -min perfusate caused prostacyclin production to reach or exceed that released during the first perfusion period. Thrombin added to the first 15-min perfusate caused both arteries and veins to release greater amounts of prostacyclin during that period.

Prostacyclin release from veins harvested after heparin administration was 50 to $100 \%$ higher than release from veins harvested prior to heparin anticoagulation. The higher release under these circumstances was statistically significant for both the thrombin-stimulated and the nonthrombin-stimulated veins $(P=0.001$ and 0.05 , respectively). Greater prostacyclin production following heparin administration did not occur with arteries.

Thromboxane release was minimal in both arteries and veins (Table 2, Figs. 3 and 4). A two- to fourfold increase in measured $\mathrm{TxB}_{2}$ occurred with arachidonic acid sup-

TABLE 1

Vessel Segment 6-keto-PGF $\alpha$ Release with and without Heparin Anticoagulation Prior to Excision

\begin{tabular}{|c|c|c|c|c|c|c|}
\hline & $015 \mathrm{~min}$ & $1530 \mathrm{~min}$ & $30-45 \mathrm{~min}$ & $45-60 \mathrm{~min}$ & $60-75 \min ^{b}$ & $P$ value $^{c}$ \\
\hline \multicolumn{7}{|l|}{ Vein } \\
\hline No heparin & $8 \pm 1$ & $4 \pm 1$ & $5 \pm 1$ & $4 \pm 1$ & $39 \pm 6$ & 0.05 \\
\hline \multicolumn{7}{|c|}{ Vein (thrombin $)^{a}$} \\
\hline No heparin & $15 \pm 3$ & $6 \pm 1$ & $5 \pm 1$ & $4 \pm 1$ & $29 \pm 5$ & \multirow[t]{2}{*}{0.001} \\
\hline Heparin & $18 \pm 4$ & $11 \pm 2$ & $8 \pm 1$ & $7 \pm 1$ & $36 \pm 7$ & \\
\hline No heparin & $81 \pm 13$ & $27 \pm 4$ & $16 \pm 3$ & $15 \pm 3$ & $118 \pm 8$ & \multirow[t]{2}{*}{0.7} \\
\hline Heparin & $77 \pm 7$ & $31 \pm 5$ & $27 \pm 7$ & $17 \pm 2$ & $114 \pm 13$ & \\
\hline \multicolumn{7}{|c|}{ Artery (thrombin) ${ }^{a}$} \\
\hline No heparin & $117 \pm 18$ & $33 \pm 5$ & $20 \pm 6$ & $14 \pm 3$ & $108 \pm 9$ & \multirow[t]{2}{*}{0.1} \\
\hline Heparin & $127 \pm 24$ & $41 \pm 9$ & $23 \pm 4$ & $15 \pm 2$ & $141 \pm 18$ & \\
\hline
\end{tabular}

Note. Data are expressed as means \pm SEM.

a Thrombin (final concentration 2 units/ml) added during first perfusion period only.

${ }^{b}$ Arachadonic acid (final concentration $4 \mu \mathrm{g} / \mathrm{ml}$ ) added during this period.

${ }^{c}$ Heparin vs no heparin groups (paired $t$ test). 
NO THROMBIN

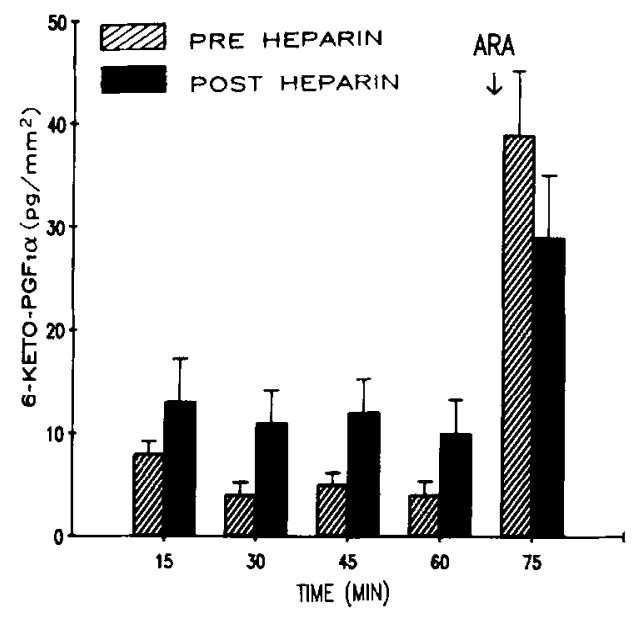

THROMBIN

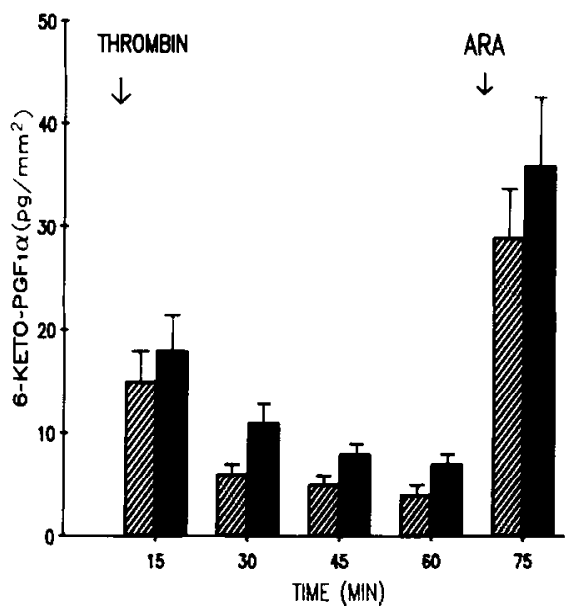

FIG. 1. Vein segment 6-keto-PGF $\alpha$ release with and without first period thrombin stimulation. Error bars represent 1 SEM. Preheparin vs postheparin; $P=0.05$ (no thrombin), $P=0.001$ (thrombin). ARA, arachidonic acid.

plementation, but $\mathrm{TxB}_{2}$ levels never exceeded $10 \%$ of the measured 6-keto- $\mathrm{PGF}_{1} \alpha$ in either arteries or veins during similar time periods. No differences existed in thromboxane release from vessels harvested before or after heparin anticoagulation.

Luminal endothelial cell coverage averaged 50 to $80 \%$ following perfusion, with the percentage coverage being similar regardless of whether vessels were harvested before or after heparin administration (Fig. 5).

\section{DISCUSSION}

In this study, heparin administered prior to vein removal resulted in higher production of prostacyclin as measured in an ex vivo perfusion system. Similar increases in prostacyclin production from arteries were not observed in the heparin group. There was no significant difference in endothelial cell coverage of vessels harvested without heparin exposure compared to those harvested after heparin administration. Since the major source of prostacyclin synthesis is endothelium, this suggests that the observed differences were actually due to higher prostacyclin production, not greater endothelial retention. However since endothelial cell coverage was determined after $e x$ vivo perfusion, it is possible that better endothelial retention did occur in the heparin group, but that the difference between groups was masked by the effects of perfusion. It is also possible that prostacyclin production is a more sensitive quantitative indicator of endothelial coverage than the relatively crude method of estimating surface coverage by scanning electron microscopic examination of random areas.

Heparin does not have a direct effect on endothelial cell prostacyclin production [10,11]. However, it is likely
NO THROMBIN

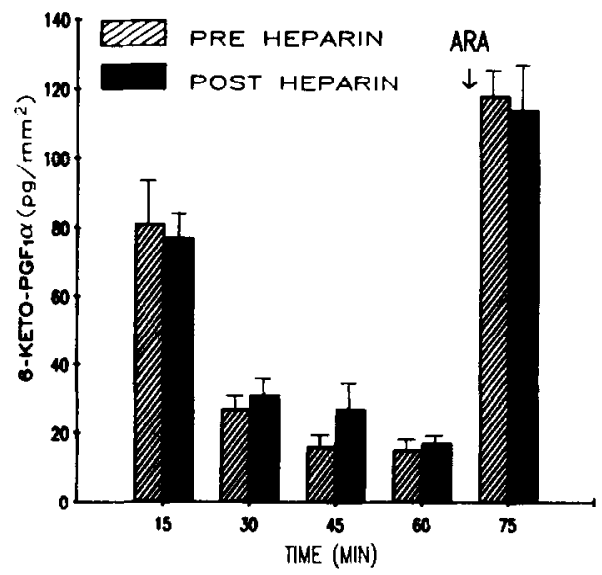

THROMBIN

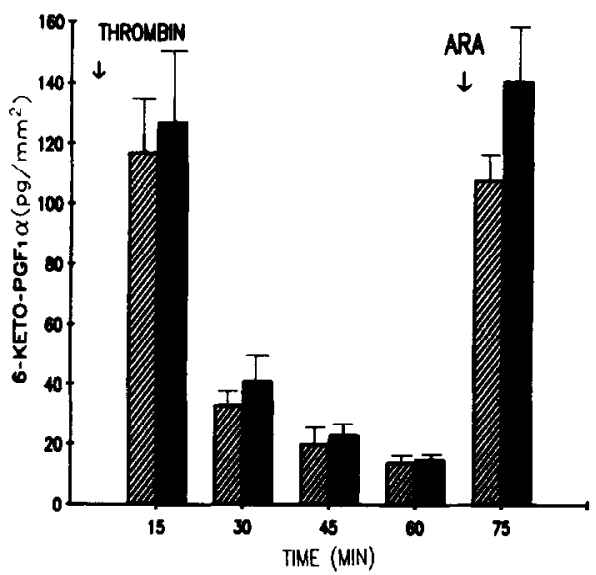

FIG. 2. Artery segment 6-keto-PGF $\alpha$ release with and without first period thrombin stimulation. Preheparin vs postheparin: $P=0.7$ (no thrombin), $P=0.1$ (thrombin). 
TABLE 2

Vessel Segment $\operatorname{TxB}_{2}$ Release with and without Heparin Anticoagulation Prior to Excision

\begin{tabular}{|c|c|c|c|c|c|c|}
\hline \multicolumn{7}{|l|}{ Vein } \\
\hline Heparin & $0.7 \pm 0.1$ & $0.3 \pm 0.1$ & $0.3 \pm 0.1$ & $0.3 \pm 0.1$ & $2.2 \pm 0.3$ & 0.5 \\
\hline \multicolumn{7}{|c|}{ Vein (thrombin) $)^{a}$} \\
\hline No heparin & $0.5 \pm 0.1$ & $0.3 \pm 0.1$ & $0.3 \pm 0.1$ & $0.2 \pm 0.1$ & $1.9 \pm 0.3$ & 0.5 \\
\hline \multicolumn{7}{|l|}{ Artery } \\
\hline No heparin & $1.0 \pm 0.1$ & $0.8 \pm 0.1$ & $0.6 \pm 0.1$ & $0.6 \pm 0.1$ & $4.0 \pm 0.5$ & \multirow[t]{2}{*}{0.9} \\
\hline Heparin & $1.7 \pm 0.5$ & $0.8 \pm 0.2$ & $0.4 \pm 0.1$ & $0.6 \pm 0.1$ & $3.9 \pm 0.4$ & \\
\hline \multicolumn{7}{|c|}{ Artery (thrombin) ${ }^{a}$} \\
\hline No heparin & $1.6 \pm 0.2$ & $0.9 \pm 0.2$ & $0.7 \pm 0.1$ & $0.6 \pm 0.1$ & $3.9 \pm 0.4$ & \multirow[t]{2}{*}{0.5} \\
\hline Heparin & $1.8 \pm 0.3$ & $0.8 \pm 0.2$ & $0.5 \pm 0.1$ & $0.5 \pm 0.1$ & $3.6 \pm 0.3$ & \\
\hline
\end{tabular}

Note. Data are expressed as means \pm SEM.

a Thrombin (firal concentration 2 units $/ \mathrm{ml}$ ) added during first perfusion period only.

${ }^{b}$ Arachadonic acid (final concentration $4 \mu \mathrm{g} / \mathrm{ml}$ ) added during this period.

c Heparin vs no heparin groups (paired $t$ test).

that the effect of thrombin generated due to the mechanical trauma of harvesting blood vessels is lessened by heparin administration, and this antithrombin activity accounts for increased prostacyclin production.

Lower and less variable prostacyclin production from rabbit aorta and vena cava segments in animals heparinized prior to vessel harvest has been reported by others [10]. The results of this latter study may appear to be the opposite of the current investigation, yet in fact these findings may be consistent with our observations, in that the former studies involved assessment of prostacyclin production immediately after vessel harvest and without a perfusion stimulus. In the current study, the 20 - to $40-$ min lag between vessel procurement and onset of perfu- sion, along with the flushing of vessels to eliminate prostacyclin produced in vivo and during the mounting, should have minimized the residual effects of in vivo stimuli. Endothelial cells exposed to thrombin are known to become refractory to further thrombin-stimulated release of prostacyclin $[12,13]$. Prostacyclin production by vein segments not exposed to heparin may reflect a refractory state induced by thrombin stimulation during the harvest process. Prior heparin exposure may protect the vessel from the effects of thrombin.

The increase in prostacyclin production with arachidonic acid supplementation is consistent with other studies that demonstrated a response to arachidonic acid after exhaustion of the release response to thrombin $[13,14]$.
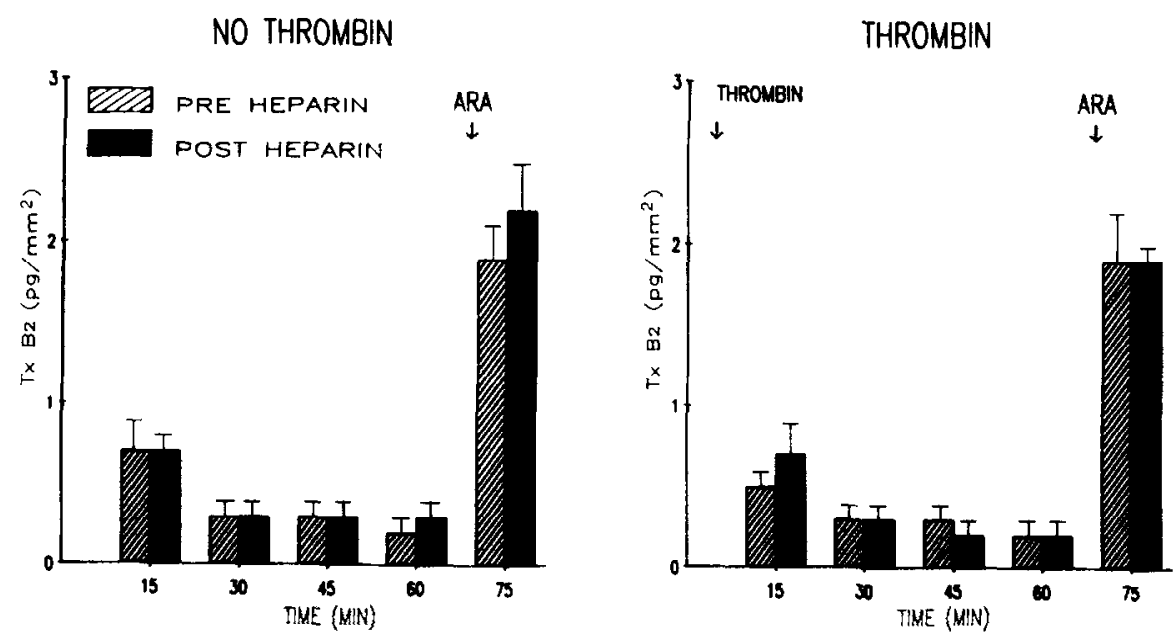

FIG. 3. Vein segment $T \times B_{2}$ release with and without first period thrombin stimulation. Preheparin vs postheparin: $P=0.5$ (no thrombin), $P=0.5$ (thrombin). 

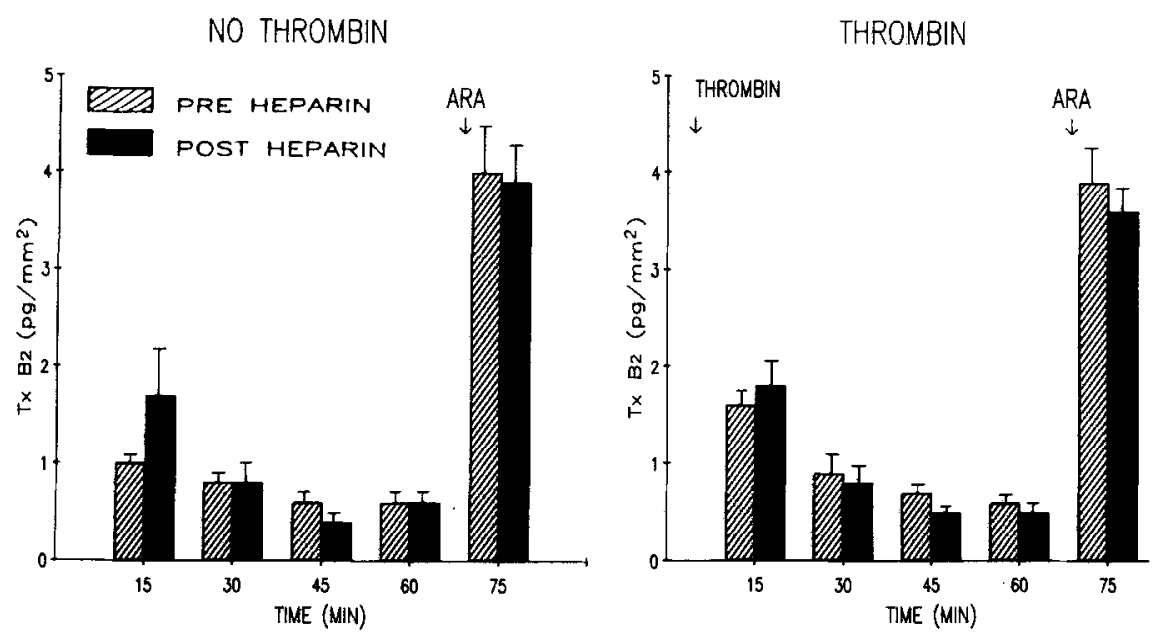

FIG. 4. Artery segment $\mathrm{TxB}_{2}$ release with and without first period thrombin stimulation. Preheparin vs postheparin: $P=0.9$ (no thrombin), $P=0.5$ (thrombin).

It has been suggested that the stimulatory effect of thrombin on prostacyclin production is mediated through increasing release of endogenous arachidonic acid from membrane phospholipids and that the refractoriness to further thrombin stimulation is due to a block at this level [14]. If the lower prostacyclin production in the noheparin group is due to a block at the level of endogenous arachidonate release, this block would be bypassed by exogenous arachidonic acid administration.

The apparent lack of a protective effect of heparin on arterial prostacyclin production may be due to differences in arterial and venous endothelium. Arterial prostacyclin production is known to be greater than venous production, given the same endothelial cell surface area $[15,16]$. Others have shown canine artery and vein to differ in endothelial cell response to thrombin, with thrombin causing endothelial-dependent arterial relaxation, but endothelialdependent venous contraction [17]. Differences have also been noted in the endothelial cell prostacyclin release following thrombin exposure, depending on the source of endothelium $[18,19]$. In this regard, thrombin generation during vessel harvest may have less of an effect on arterial than venous endothelium.

The clinical implications of this study are undefined. It is likely that the advantages of vein grafts over prosthetic grafts in lower extremity reconstructions is due at least in part to the thromboresistant nature of endothelium, of which prostacyclin production is an important component. Better functional preservation of the endothelial cell capacity for prostacyclin production may reduce early platelet deposition after placement of vein grafts in the arterial circulation. In this regard, administration of heparin prior to beginning dissection of a vein intended for use as an arterial substitute may reduce certain potentially harmful effects of thrombin generated during the dissection process.

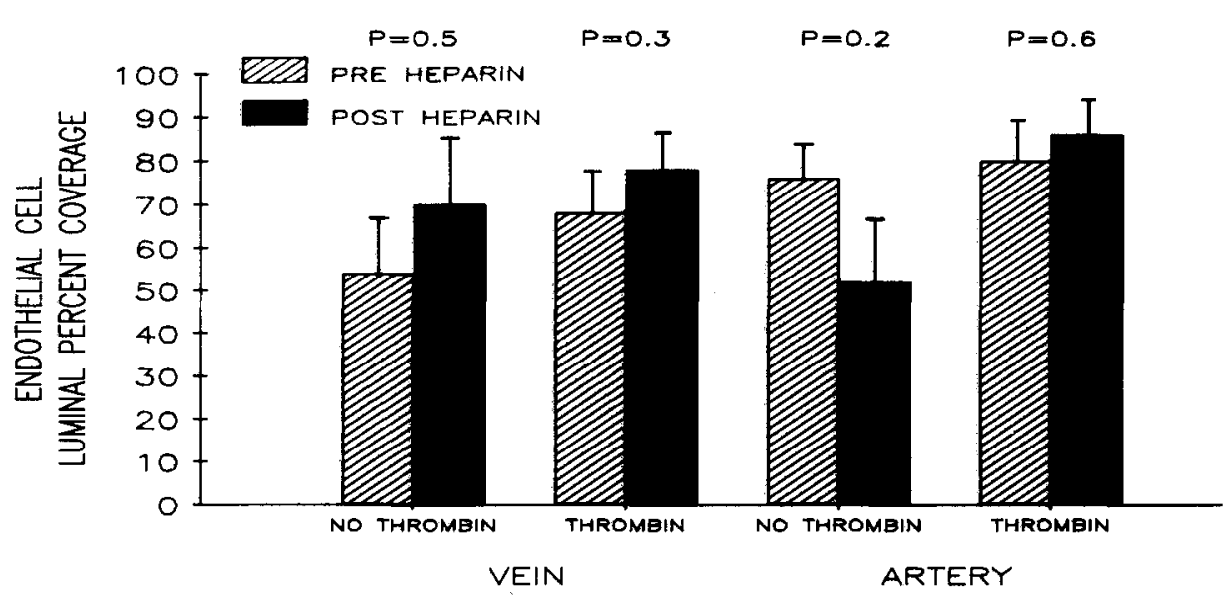

FIG. 5. Luminal surface area coverage by endothelium after perfusion as determined by scanning electron microscopy. 


\section{REFERENCES}

1. Mehta, J., and Roberts, A. Human vascular tissues produce thromboxane as well as prostacyclin. Amer. J. Physiol. 244: R839, 1983.

2. Ingerman-Wojenski, C., Silver, M. J., Smith, J. B., and Macarack, E. Bovine endothelial cells in culture produce thromboxane as well as prostacyclin. J. Clin. Invest. 67: 1292, 1981.

3. Adcock, O. T., Jr., Adcock, G. I. D., Wheeler, J. R., Gregory, R. T., Snyder, S. O., and Gayle, R. G. Optimal techniques for harvesting and preparation of reversed autogenous vein grafts for use as arterial substitutes: A review. Surgery 96(5): 886, 1984.

4. Owen, W. G., Goeken, I. A., and Lollar, P. Thrombin-endothelial interactions. In M. H. Gimbrone Jr. (Ed.), Vascular Endothelium in Hemostasis and Thrombosis. New York: Churchill Livingstone, 1986. P. 57.

5. Killackey, J. J. F., Johnston, M. G., and Movat, H. Z. Increased permeability of microcarrier-cultured endothelial monolayers in response to histamine and thrombin: A model for the in vitro study of increased vasopermeability. Amer. J. Pathol. 122(1): 50, 1986.

6. Hladovec, J. The effect of heparin on endothelial stability. Thromb. Res. 35: 347, 1984.

7. Gertz, S. D., Rennels, M. L., and Nelson, E. Endothelial cell ischemic injury: Protective effect of heparin or aspirin assessed by scanning electron microscopy. Stroke 6: 357, 1975.

8. Brunkwall, J. S., Stanley, J. C., Graham, L. M., and Burkel, W. E. Influence of pressure, flow rate, and pulsatility on release of 6-keto $\mathrm{PGF}_{1} \alpha$ and $\mathrm{TxB}_{2}$ in ex vivo perfused canine veins. $J$. Vasc. Surg. 7: 99-107, 1988.

9. Simmons, P. M., Salmon, J. A., and Moncada, S. The release of leukotriene $B_{4}$ during experimental inflammation. Biochem. Pharmacol. 32: 1353, 1983.

10. Buchanan, M. R., Dejana, E., Cazenave, J. P., Mustard, J. F., and Hirsh, J. Uncontrolled $\mathrm{PGI}_{2}$ production by whole vessel wall segments due to thrombin generation in vivo and its prevention by heparin. Thromb. Res. 16: 551, 1979.
11. Roncaglioni, M. C., Di Minno, G., De Gaetano, G., and Donati, M. B. Increased $P G I_{2}$ activity in venous tissues from rats on oral contraceptives is not prevented by heparin administration before vessel isolation. Thromb. Res. 18: 895, 1980.

12. DeGroot, P. G., Brinkman, H. J. M., Gonsalves, M. D., and Van Mourik, J. A. The role of thrombin in the regulation of the endothelial prostaglandin production. Biochim. Biophys. Acta 846: $342,1985$.

13. Dejana, E., Balconi, G., DeCastellarnau, C., Barbieri, B., et al. Prostacyclin production by human endothelial and bovine smooth muscle cells in culture. Effect of repeated stimulation with arachidonic acid, thrombin and ionophore A23187. Biochim. Biophys. Acta 750: 261, 1983.

14. Weksler, B. B., Ley, C. W., and Jaffe, E. A. Stimulation of endothelial cell prostacyclin production by thrombin, trypsin, and the ionophore A23187. J. Clin. Invest. 62: 923, 1978.

15. Subramanian, V. A., Hernandez, Y., Tack-Goldman, K., Grabowski, E. F., and Weksler, B. B. Prostacyclin production by internal mammary artery as a factor in coronary artery bypass grafts. Surgery 100(2): 376, 1986.

16. Chaikhouni, A., Crawford, F. A., Kochel, P. J., Olanoff, L. S., and Halushka, P. V. Human internal mammary artery produces more prostacyclin than saphenous vein. $J$. Thorac. Cardiovasc. Surg. 92: $88,1986$.

17. De Mey, J. G., and Vanhoutte, P. M. Heterogeneous behavior of the canine arterial and venous wall: Importance of the endothelium. Circ. Res. 51(4): 439, 1982.

18. Goldsmith, J. C., and Kisker, C. T. Thrombin-endothelial cell interactions: Critical importance of endothelial cell vessel of origin. Thromb. Res. 25: 131, 1982.

19. Goldsmith, J. C., Jafvert, C. T., Lollar, P., Owen, W. G., and Hoak, J. C. Prostacyclin release from cultured and ex vivo bovine vascular endothelium: Studies with thrombin, arachidonic acid, and ionophore A23187. Lab. Invest. 45(2): 191, 1981. 